\title{
Kernos
}

Revue internationale et pluridisciplinaire de religion grecque antique

13 | 2000

Varia

\section{Marie-Françoise BASLEZ, Bible et histoire. Judaïsme, hellénisme, christianisme}

\section{André Motte}

Édition électronique
URL : http://journals.openedition.org/kernos/1336

DOI : 10.4000/kernos. 1336

ISSN : 2034-7871

\section{Éditeur}

Centre international d'étude de la religion grecque antique

Édition imprimée

Date de publication : 1 janvier 2000

ISSN : 0776-3824

\section{Référence électronique}

André Motte, « Marie-Françoise Bastez, Bible et histoire. Judaïsme, hellénisme, christianisme », Kernos [En ligne], 13 | 2000, mis en ligne le 21 avril 2011, consulté le 24 septembre 2020. URL : http:// journals.openedition.org/kernos/1336; DOI : https://doi.org/10.4000/kernos.1336 
l'occurrence navale; on peut postuler un archétype pour Or. Sib. III et Antisthène, connu aussi par « Hystaspe » (Loreto, p. 443-486; bonne méthodologie). L'oracle sibyllin prédisant l'éruption du Vésuve (79 ap. J.-C.) chez Plutarque (398e et 566e) était d'origine juive et n'est pas émis ex eventu (Brenk, p. 487-501).

IV. Interpretazioni e trasformazioni: gli Oracula nella cultura cristiana. Tout un réseau de textes nous permet de tracer la voie qui conduit à l'adoption chrétienne de la Sibylle et d'entrevoir les raisons de ce choix (Sfameni-Gasparro, p. 505-553; étude remarquable). Procope, dans sa Guerre Gotbique, a recours à deux oracles sibyllins catastrophiques pour introduire une dose de dramatisation dans son récit, ce qui montre la force de cette tradition au $\mathrm{vI}^{\mathbf{e}}$ siècle ap. J.-C. (Pricoco, p. 555-567). La Sibylle subi au Moyen Âge une évolution qui s'explique par la polarisation de l'orientation hagiographique : de prophétesse elle devient sorcière (Scalera McClintock, p. 569-579). Mais sa sagesse a fait d'elle la candidate favorite pour proclamer la révélation chrétienne, tel qu'on le constate depuis l'école d'Alexandrie (Sardella, p. 581-602). Un nouveau texte de la Newberry Library vient enrichir la tradition de la Sibylle Tiburtine (McGinn, p. 603-644; excellente contribution).

V. La tradizione: Sibille e Sibilla, le profetesse et la regina. - Dans le cadre de la géographie "sibylline" des monts picéniens homonymes, trois épisodes des empereurs Vitellius, Claude II le Gothique et Aurélien servent à témoigner de l'antiquité des consultations dans la grotte de la Sibylle dans le monde ancien (†Allevi, p. 647-671). Le recours à la fata Sibylla par Antoine de la Sale dans La Salade renferme un lecture de la culture de son temps et une réflexion sur la fragilité des croyances (Böhler-Regnier, p. 673-693). Le paradis sibyllin « demonisé », plein d'éléments partagés avec quelques autres légendes du Moyen Âge, présente des différences remarquables, mais aussi des points en commun, dans Le paradis de la reine Sibylle, d'Antoine de la Sale, et dans le Guerrin Meschino (Montesano, p. 695-708). On aperçoit une correspondance entre les textes sibyllins des XIVe et $\mathrm{xv}^{\mathrm{e}}$ siècles et l'évolution iconographique de la Sibylle en Italie (Castelli, p. 709739).

VI. La Sibilla e il suo territorio. - Les traces du paganisme persistent avec force en Italie même pendant le $\mathrm{IV}^{\mathrm{e}}$ siècle ap. J.-C. (Paci, p. 743-753). Les pouvoirs magiques et les prodiges de Sainte Rita di Cascia appartiennent à la typologie sibylline, bien enracinée dans sa terre natale (Scaraffia, p. 755-771). La tradition de la Sibilla Porrina, liée à celle de Sainte Rita, renforce la proximité des deux typologies (Giacalone, p. 773-802). Et, pour terminer, un parcours dans les différents paysages sibyllins de l'Italie accompagne la richesse de ses traditions (Di Modugno, p. 803-822; carte, p. 822).

À regretter l'absence de tout index (sauf le général, p. 7-9) et les nombreuses coquilles et erreurs formelles (y compris des altérations dans l'ordre des paragraphes aux p. 159 et 185) dont je renonce à donner le détail. Mais le bilan est sans doute très positif. Ce livre est d'ores et déjà un volume indispensable dans la bibliographie sibylline, non seulement dans le domaine strict du prophétisme, mais aussi pour la connaissance de la tradition culturelle occidentale.

Emilio Suárez de la Torre (Universidad de Valladolid)

Marie-Françoise BASLEz, Bible et histoire. Judaïsme, hellénisme, christianisme, Paris, Fayard, 1998. 1 vol. $15 \times 23,5$ cm, 479 p. ISBN : 2-213-60202-6.

Un « essai », annonce modestement l'auteur dans son avant-propos. On a tôt fait cependant de découvrir que l'essai en question a toutes les qualités d'un 
grand livre. C'est qu'il est l'œuvre d'une historienne très expérimentée, sachant poser les bonnes questions, rompue à l'exploitation critique des sources anciennes et très attentive aux contextes politiques, sociaux et culturels dans sa façon d'enquêter sur ce sujet religieux. L'étude a du reste été précédée d'une série de travaux remarqués sur l'histoire politique du monde grec antique (1994), sur le thème du voyage (1993) et de l'étranger (1984) dans l'Antiquité et sur saint Paul (1991), sans compter une thèse de doctorat qui portait sur Les conditions de pénétration et de diffusion des religions orientales à Délos (1977). L'A. est donc particulièrement familiarisée avec les problèmes d'acculturation et de résistance identitaire auxquels le présent ouvrage réserve une attention constante.

Comme l'indique le sous-titre, la Bible est prise ici dans sa double composante, juive et chrétienne, la période étudiée débutant à l'époque hellénistique pour prendre fin au début du II $^{\mathrm{e}}$ siècle de notre ère. L'exploration est centrée, chaque fois qu'il est possible, sur des hommes, - figures individuelles et communautés locales, soit qu'ils aient enrichi le Livre de créations nouvelles, lesquelles sont nombreuses à cette époque, soit qu'ils se réclament de son message, soit encore qu'ils se soient à ce titre opposés les uns aux autres ou qu'ils aient eu à se défendre contre des adversaires extérieurs. L'hellénisme est présent presque à chaque page, tantôt en toile de fond, précieux bien souvent pour éclairer telle ou telle donnée biblique, tantôt à l'avant-plan comme enjeu de conflits ouverts. Et plus avant, c'est aussi la romanitas qui devient une référence obligée. Loin de se cantonner à Jérusalem et à la Palestine, le parcours suivi accompagne plus d'une fois les Juifs de la Diaspora méditerranéenne et aussi les apôtres chrétiens dans leurs lointaines randonnées missionnaires.

Tout commence d'ailleurs, si l'on peut dire, à Alexandrie, la ville où a été entreprise, avec l'appui de Ptolémée, la traduction en grec de la Bible par les «Septante ", un événement d'une importance considérable, souligne l'A., pour l'histoire religieuse et l'histoire culturelle. Tel est l'objet du premier chapitre de l'ouvrage, lequel se présente non comme une histoire systématique du développement et de la diffusion des écrits vétéro- et néo-testamentaires, mais comme une succession d'enquêtes portant chacune sur une question particulière. Sont longuement examinées, par exemple, les relations parfois difficiles entre le « Temple et l'État grec », représenté d'abord par le pouvoir des Séleucides. Celui-ci, particulièrement en la personne d'Antiochos IV, manifeste une volonté de déjudaïsation qui entraînera l'insurrection des Maccabées contre les Juifs hellénisés; la révolte se solde par une acculturation accélérée des résistants et sera suivie d'une intense création littéraire, dans les genres héroïque, mystique et apocalypti que. Le culte royal et l'idolâtrie sont aussi, à l'époque, des causes d'affrontement. Plus tard, dans la Diaspora, l'auteur du Livre de Salomon, un ouvrage écrit en grec, focalisera sa critique sur le dionysisme en même temps qu'il dénonce l'antisémitisme alexandrin.

Un chapitre est aussi réservé à la découverte passionnante des manuscrits de Qumrân, que l'A. présente comme un véritable «miroir de l'histoire et de la religiosité juives ", résistant ainsi aux essais trop sommaires d'identification à une secte, alors que Qumrân révèle des comportements pluriels comme il confirme aussi le caractère pluriel de la Bible. Après une enquête bienvenue, de type sociologique, sur le milieu où a vécu Jésus et que marquent divers clivages géographiques, religieux et culturels, l'ouvrage entreprend d'esquisser une " histoire en rétrospective » qui va du Christ à Jésus. Il s'agit, en effet, pour l'historien, d'être attentif au fait que les évangiles sont un acte de foi, qu'ils révèlent plus qu'ils ne racontent et que la signification de l'événement relaté prend donc le pas sur sa réalité. De façon générale, dans l'Antiquité, le genre biographique est davantage 
démonstratif que documentaire, mais l'historien rencontre ici des difficultés spécifiques. Après une introduction méthodologique appropriée, l'A. aborde avec maîtrise une série de problèmes délicats qui vont du recensement ( un événement reconstruit 》), censé avoir commandé le voyage de Marie et Joseph, au procès de Jésus (celui de Socrate apparaît quelquefois en filigrane dans les témoignages) et à la crucifixion en passant notamment par le thème des généalogies, de la naissance miraculeuse, de la thaumaturgie et du miracle. Abordant des questions non moins délicates, le chapitre consacré à l'Église de la Pentecôte nous vaut d'intéressantes considérations sur l'inspiration et la communication, l'occasion de faire apparaître à la fois des ressemblances et des différences entre les sensibilités grecque et chrétienne, à propos, par exemple, des visions en songe, des apparitions et des phénomènes d'extase.

Les sections suivantes, après un arrêt sur l'Église de Jérusalem, ne sont pas moins passionnantes et riches de perspectives nouvelles. Pour faire bref, disons seulement qu'elles s'attachent à l'examen de problèmes liés à la pénétration et à la diffusion du christianisme dans le monde grec, avec une attention spéciảle accordée aux difficultés d'intégration et aux crises identitaires (e.g. : l'apparition de l'hérésie, la question des «faux prophètes », la magie, le culte impérial) ainsi qu'aux rapports entre les chrétiens et le pouvoir (en particulier, la difficile question des persécutions et de leur fondement juridique).

L'ouvrage se lit d'un bout à l'autre avec un intérêt soutenu. À défaut de la compétence nẹcessaire pour juger de certaines positions de fond, on ne peut manquer d'apprécier l'esprit critique et la rigueur scientifique qui président à toutes les enquêtes. L'ouvrage s'adressant à un large public cultivé, on comprend que l'appareil justificatif se limite le plus souvent, dans les notes, à de simples références aux sources anciennes, mais il convient d'attirer l'attention sur la très riche bibliographie commentée qui figure à la fin de l'ouvrage (p. 405-451) et qui suit l'ordre des onze chapitres. S'y ajoutent un relevé des sources, un index des noms de lieux et un autre des noms de personnes, une table des neuf cartes, fort bienvenues, disséminées dans le livre ainsi qu'une table des matières détaillée. La présentation, on le devine, est à tous égards très soignée et elle contribue, avec le style sobre et clair, à l'agrément de la lecture.

André Motte

(Université de Liège)

Françoise Mies (éd.), Toute la sagesse du monde. Hommage à Maurice Gilbert, s.j. Pour le $65^{e}$ anniversaire de l'exégète et du recteur, Namur, Presses universitaires, 1999.1 vol. $14,5 \times 20,5 \mathrm{~cm}, 768$ p. (Connaître et croire, 4). ISBN : 2-87037-278-7.

Le R.P. M. Gilbert vient de quitter la fonction de recteur des Facultés universitaires Notre-Dame de la Paix de Namur qu'il exerçait depuis 1993, après avoir été auparavant recteur des Instituts Bibliques de Rome (1978-1984) et de Jérusalem (1984-1992), deux institutions dans lesquelles il a repris actuellement son enseignement. Sa très copieuse bibliographie, que détaille le volume (p. 705-731), témoigne non seulement d'une compétence particulièrement avérée dans le domaine biblique, mais aussi d'une fine connaissance de l'hellénisme. Méritent notamment d'être épinglés à cet égard sa thèse de doctorat : La critique des dieux dans le Livre de la Sagesse, Rome, 1973, ainsi qu'un de ses tout derniers articles: La sagesse de Salomon et l'bellénisme, dans Hieros. Bulletin de la Société belgoluxembourgeoise d'bistoire des religions, 4 (1999), p. 11-31. Les livres sapientiaux ayant été son sujet de prédilection (à noter, en particulier, cet important ouvrage collectif dont il est l'éditeur scientifique : La sagesse de l'Ancien Testament, 